\title{
Evaluation of a curcumin analog as an anti-cancer agent inducing ER stress-mediated apoptosis in non-small cell lung cancer cells
}

Zhiguo Liu ${ }^{1 \dagger}$, Yusheng Sun ${ }^{2 \dagger}$, Luqing Ren ${ }^{1}$, Yi Huang ${ }^{1}$, Yuepiao Cai ${ }^{1}$, Qiaoyou Weng ${ }^{1}$, Xueqian Shen ${ }^{1}$, Xiaokun Li ${ }^{1}$, Guang Liang ${ }^{1 *}$ and Yi Wang ${ }^{1 *}$

\begin{abstract}
Background: Recent advances have highlighted the importance of the endoplasmic reticulum (ER) in cell death processes. Pharmacological interventions that effectively enhance tumor cell death through activating ER stress have attracted a great deal of attention for anti-cancer therapy.

Methods: A bio-evaluation on 113 curcumin analogs against four cancer cell lines was performed through MTT assay. Furthermore, real time cell assay and flow cytometer were used to evaluate the apoptotic induction of (1E,4E)-1,5-bis(5-bromo-2-ethoxyphenyl)penta-1,4-dien-3-one (B82). Western blot, RT-qPCR, and siRNA were then utilized to confirm whether B82-induced apoptosis is mediated through activating ER stress pathway. Finally, the in vivo anti-tumor effect of B82 was evaluated.

Results: B82 exhibited strong anti-tumor activity in non-small cell lung cancer (NSCLC) H460 cells. Treatment with B82 significantly induced apoptosis in $\mathrm{H} 460$ cells in vitro and inhibited $\mathrm{H} 460$ tumor growth in vivo. Further studies demonstrated that the B82-induced apoptosis is mediated by activating ER stress both in vitro and in vivo.

Conclusions: A new monocarbonyl analog of curcumin, B82, exhibited anti-tumor effects on H460 cells via an ER stress-mediated mechanism. B82 could be further explored as a potential anticancer agent for the treatment of NSCLC.
\end{abstract}

Keywords: Curcumin analogs, Anti-cancer, ER stress, Non-small cell lung cancer, CHOP

\section{Background}

Recent advances have highlighted the importance of the endoplasmic reticulum (ER) in cell death processes. Perturbation of ER functions leads to ER stress, which has been previously associated with a broad variety of diseases, while prolonged ER stress can activate apoptotic pathways in damaged cells [1]. For this reason, pharmacological interventions that effectively enhance tumor cell death through activating ER stress have attracted a great deal of attention for anti-cancer therapy.

Curcumin is an active phenolic compound extracted from the rhizome of the plant Curcuma longa. Extensive

\footnotetext{
*Correspondence: wzmcliangguang@163.com; yi.wang1122@gmail.com ${ }^{\dagger}$ Equal contributors

${ }^{1}$ Chemical Biology Research Center, School of Pharmaceutical Sciences, Wenzhou Medical University, University Town, 325035 Wenzhou, Zhejiang, China
} Full list of author information is available at the end of the article research over the last half century has revealed various bio-functions of curcumin. Its anti-cancer effect has been seen in a few clinical trials, mainly as a native chemoprevention agent in colon and pancreatic cancer [2]. Recently, it was reported that curcumin exerts its pro-apoptotic effects by inducing ER stress in several tumor cells, including acute promyelocytic leukemia cells [3], human non-small cell lung cancer H460 cells [4], and human liposarcoma cells [5]. Although curcumin has an evident anti-cancer activity, rapid metabolism and low bioavailability have been highlighted as the major limitations in therapeutic applications [6]. To enhance metabolic stability and pharmacological potency, various curcumin analogs have been synthesized, among which, the mono-carbonyl analogs of curcumin (MACs) have been developed by our laboratory in the past six years. Without the central $\beta$-diketone moiety in curcumin structure, the MACs exhibit

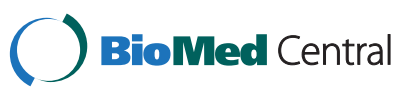


enhanced stability in vitro and an improved pharmacokinetic profile in vivo [7-9].

Advance in molecular biology has allowed a change in anti-cancer therapy trends, from classic cytotoxic strategies to the development of new therapies which target the special apoptosis response in tumor cells. The aim of our laboratory is to find anti-cancer therapeutic agents with relatively new mechanism. In continuation of our ongoing research, we evaluated here 113 synthetic MACs for their anti-proliferative effects, among which, the active compound (1E,4E)-1,5-bis(5-bromo-2-ethoxyphenyl)penta1,4-dien-3-one (B82) was further examined as an excellent anti-tumor agent both in vitro and in vivo. Importantly, our results showed that B82 may induce cancer cell apoptosis via activating ER stress-mediated apoptotic pathway.

\section{Methods}

\section{Cell lines and reagents}

Human breast cancer cell line MCF-7, carcinomic human alveolar basal epithelial cell line A549, human lung carcinoma cell line H460, human liver carcinoma cell line HepG2, and normal human lung (bronchial) epithelial cell line BEAS-2B were purchased from ATCC (Manassas, VA); normal human liver cell line HL-7702 was purchased from Shanghai Institute of Life Sciences Cell Resource Center (Shanghai, China). The cells were cultured in RPMI 1640 medium (Invitrogen, Carlsbad, $C A$ ) supplemented with 5\% heat-inactivated FBS (Atlanta Biologicals Inc., Lawrenceville, GA) and $100 \mathrm{U} / \mathrm{mL}$ penicillin and streptomycin (Mediatech Inc., Manassas, VA), and incubated at $37^{\circ} \mathrm{C}$ with $5 \% \mathrm{CO}_{2}$. FITC Annexin V apoptosis Detection Kit I was purchased from BD Pharmingen (Franklin Lakes, $N J$ ). Anti-CHOP, anti-GRP 78, anti-GAPDH, anti-Actin, anti-Bcl-2, anti-Cyclin D1, anti-COX-2, goat anti-rabbit IgG-HRP, mouse anti-goat IgG-HRP antibodies were from Santa Cruz Biotechnology (Santa Cruz, CA), and anti-cleavaged caspase-3 was from Cell Signaling Technology (Danvers, $M A$ ). Ambion RNAqueous kit was purchased from Applied Biosystems Inc. (Foster City, CA). Caspase 3 Activity Assay Kit was from Beyotime Biotech (Nantong, China).

\section{Chemistry}

Curcumin was purchased from Sigma (St. Louis, MO). Curcumin analogues 1-113 were synthesized by our laboratory, and were reported in our previous articles with their anti-inflammatory activities [9-12]. The names and structures of these compounds were shown in Additional file 1: Table S1. Before used to the biological experiments, compounds were purified by re-crystallization or silica gel chromatography to reach the purity higher than $97.0 \%$. In in vitro experiments, compounds were used in DMSO solution, when the final concentration of DMSO in cultural medium is $0.1 \%$.

\section{Methyl thiazolyl tetrazolium (MTT) assay}

All experiments were carried out $24 \mathrm{~h}$ after cells were seeded. Tested compounds were dissolved in DMSO and diluted with 1640 medium to final concentrations of 0.3 , $1.25,5,20$, and $80 \mu \mathrm{M}$. The tumor cells were incubated with test compounds for $72 \mathrm{~h}$ before the MTT assay. Curcumin was applied as the positive control.

\section{Dynamic monitoring of $\mathrm{H} 460$ cell proliferation using the RT-CES system}

The real time cell electronic sensing assay is based on electrical impedance readings in cell monolayers plated in wells containing built-in gold electrodes. We have used the ACEA RT-CES analyzer, 8 well e-plates, and the integrated software from Acea Biosciences Inc. (San Diego, CA). Cells were plated at a density of 30,000 cells/well in $100 \mu \mathrm{l}$ of medium. The analyzer and the installed plates were placed in a standard cell culture incubator, at $37^{\circ} \mathrm{C}$ in a humidified atmosphere of $5 \% \mathrm{CO}_{2}$. Cells were allowed to adhere to plates overnight. After cell seeded, the analyzer was programmed to take readings during $0-96 \mathrm{~h}$; and B82 at 2.5 or $10 \mu \mathrm{M}$ was added to the medium at $40 \mathrm{~h}$ after incubation. Data were recorded and analyzed using the integrated software. The cell index is a quantitative measure of the spreading and/or proliferative status of the cells in an electrode-containing well.

\section{Cell apoptosis analysis}

$\mathrm{H} 460$ cells were placed in $60-\mathrm{mm}$ plates for $12 \mathrm{~h}$, and then treated with varying doses $(2.5,5$ and $10 \mu \mathrm{M})$ of compound B82, curcumin $(10 \mu \mathrm{M})$ or vehicle (DMSO, $3 \mu \mathrm{L}$ ) for $12 \mathrm{~h}$. Cells were then harvested and stained with Annexin $\mathrm{V}$ and propidium iodide (PI) in the presence of $100 \mathrm{mg} / \mathrm{mL}$ RNAse and $0.1 \%$ Triton X-100 for $30 \mathrm{~min}$ at $37^{\circ} \mathrm{C}$. Flow-cytometric analysis was performed using FACScalibor $(B D, C A)$.

\section{Western blot analysis}

Cells or homogenated tumor tissues were lysated. The protein concentrations in all samples were determined by using the Bradford protein assay kit (Bio-Rad, Hercules, $C A$ ). Lysates were then analyzed through western blot assay, and the immunoreactive bands were visualized by using ECL kit (Bio-Rad, Hercules, CA).

\section{RNA isolation and real-time quantitative PCR}

Total mRNA was isolated from the treated cells using Ambion RNAqueous kit after treatment with compounds or control DMSO. The High-Capacity cDNA Archive Kit was used to obtain first-strand cDNAs of mRNAs. The mRNA levels of CHOP, XBP-1, ATF-4 and GRP78 were quantified by specific gene expression assay kits and primers on iQ5 Multicolor real-time PCR detection 
system (Bio-Rad, Hercules, $C A$ ) and normalized to internal control $\beta$-actin mRNA.

\section{Caspase- 3 activation assay}

Caspase- 3 activity was determined using a Caspase-3 activity kit (Beyotime institute of biotechonoly, Nantong, China) according to the manufacturer's protocol. The OD value representing caspase- 3 activity was detected with a microplate spectrophotometer $(M D$, Sunnyvale, $C A)$ at $405 \mathrm{~nm}$. The caspase- 3 activity was normalized by the protein concentration of the corresponding cell lysate, and was expressed in enzymatic units per mg of protein.

\section{Construction of lentiviral siRNA for CHOP}

The sense sequence of the siRNA cassettes specifically targeting the nucleotides of $\mathrm{CHOP}$ was designed through siRNA Target Finder (Ambion, Austin, TX). A two-step polymerase chain reaction (PCR) strategy was performed using two separate reverse primers to generate a siRNA expression cassette (SEC) consisting of human U6 promoter and a hairpin siRNA cassette plus terminator and subcloned into pGL3.7 vector, which encodes the CMV-promoted EGFP (enhanced green fluorescent protein) marker as internal control. The resulting lentiviral siRNA vector was confirmed by restriction enzyme digestion and DNA sequencing. The sequence of CHOP siRNA is $5^{\prime}$-GCAGG AAATCGAGCGCCTGAC-3'. The recombinant lentiviruses were produced by transient transfection of $\mathrm{H} 460$ cells using FuGene 6 Transfection reagent (Roche Inc., Nutley, $N)$ ). Titers were determined by infecting $\mathrm{H} 460$ cells with serial dilutions of concentrated lentivirus and counting EGFP-expressing cells after $48 \mathrm{~h}$ under fluorescent microscopy.

\section{In vivo antitumor study}

All animal experiments complied with the Wenzhou Medical College Policy on the Care and Use of Laboratory Animals (Wenzhou Medical University Animal Policy and Welfare Committee, 201100009). Five-week-old to sixweek-old athymic nu/nu BALB/cA male mice (18-22 g) were purchased from Vital River Laboratories (Beijing, China). Animals were housed at a constant room temperature with a 12:12 hr light/dark cyclic, and fed a standard rodent diet and water. $\mathrm{H} 460$ cells were harvested, and mixed with Matri Gel in 1:1, and then injected subcutaneously into the right flank $\left(2 \times 10^{6}\right.$ cells in $200 \mu \mathrm{L}$ PBS $)$ of 7-week-old male BALB/cA nude mice. One day after injected with $\mathrm{H} 460$ cells, treated mice were intraperitoneally (i.p.) injected with a water-soluble preparation of B82 in PBS at dosage of $5 \mathrm{mg} / \mathrm{kg} /$ day for 28 days, whereas control mice were injected with liposome vehicle in PBS. The tumor volumes were determined by measuring length (l) and width $(\mathrm{w})$ and calculating volume $\left(\mathrm{V}=0.52 \times 1 \times \mathrm{w}^{2}\right)$ at the indicated time points. The tumor weights were recorded on the day of scarification.

\section{Immunohistochemistry}

The harvested tumor tissues were fixed in $10 \%$ formalin at room temperature, processed and embedded in paraffin. Parraffin-embedded tissues were sectioned ( $5 \mu \mathrm{m}$ thick). Tissue sections were primarily stained with indicated antibodies. The signal was detected by biotinylated secondary antibodies, and developed in DAB. Quantity assay of the immunochemistry data was obtained with Image-Pro Plus 6.0 (Media Cybernetics, Inc., Bethesda, MD).

\section{Statistical analysis}

All experiments were assayed in triplicate $(n=3)$. Data are expressed as means \pm SEM. All statistical analyses were performed using GraphPad Pro. Prism 5.0 (GraphPad, San Diego, $C A$ ). Student's t-test was employed to analyze the differences between sets of data. A $p$ value $<0.05$ was considered significant.

\section{Results and discussion}

Anti-tumor evaluation of 113 curcumin analogs led to the discovery of active compound B82

It has been reported that curcumin possesses a widespectrum of anti-tumor properties. Our laboratory has been engaged in finding promising anti-inflammatory and anti-cancer agents from curcumin analogs. Due to the important role of $\beta$-diketone in the metabolic defect of curcumin, we designed a series of stable MACs by deleting $\beta$-diketone moiety. To date, more than 400 MACs have been synthesized. To find the potential anti-cancer candidates from curcumin analogs, we tested the cytotoxicity of 113 MACs (shown in Figure 1A and Additional file 1: Table S1) in several tumor cell lines by MTT assay. The chemical structures and growth-inhibitory $\mathrm{IC}_{50}$ values of the best seven active compounds against H460, A549, HepG2, and MCF-7 cells were shown in Figure 1B and 1C. Among these compounds, B82 exhibited the strongest anti-tumor activity against $\mathrm{H} 460(2.02 \pm 0.32 \mu \mathrm{M})$, A549 $(2.16 \pm 1.07 \mu \mathrm{M})$, and MCF-7 cells $(2.21 \pm 0.21 \mu \mathrm{M})$ compared with other tested compounds. B82 also exhibited a strong cytotoxicity in HepG2 cells $\left(\mathrm{IC}_{50}=5.21 \pm 1.65 \mu \mathrm{M}\right)$.

The cytotoxic evaluation of these 113 compounds supported our previously published structure-activity relationship (SAR) analysis of MACs [13]. Most obviously, curcumin analogs with the acetone or cyclohexanone linker in the structures of MACs are beneficial to increasing the cytotoxic activity compared to the cyclopentanone linker. We also found that, in the chalcone-structure-containing MACs, the cytotoxic activity of compounds could be increased by the electron-donating substituents in both rings, especially at 4-position of ring $A$, and could be reduced through the induction of electron-withdrawing 


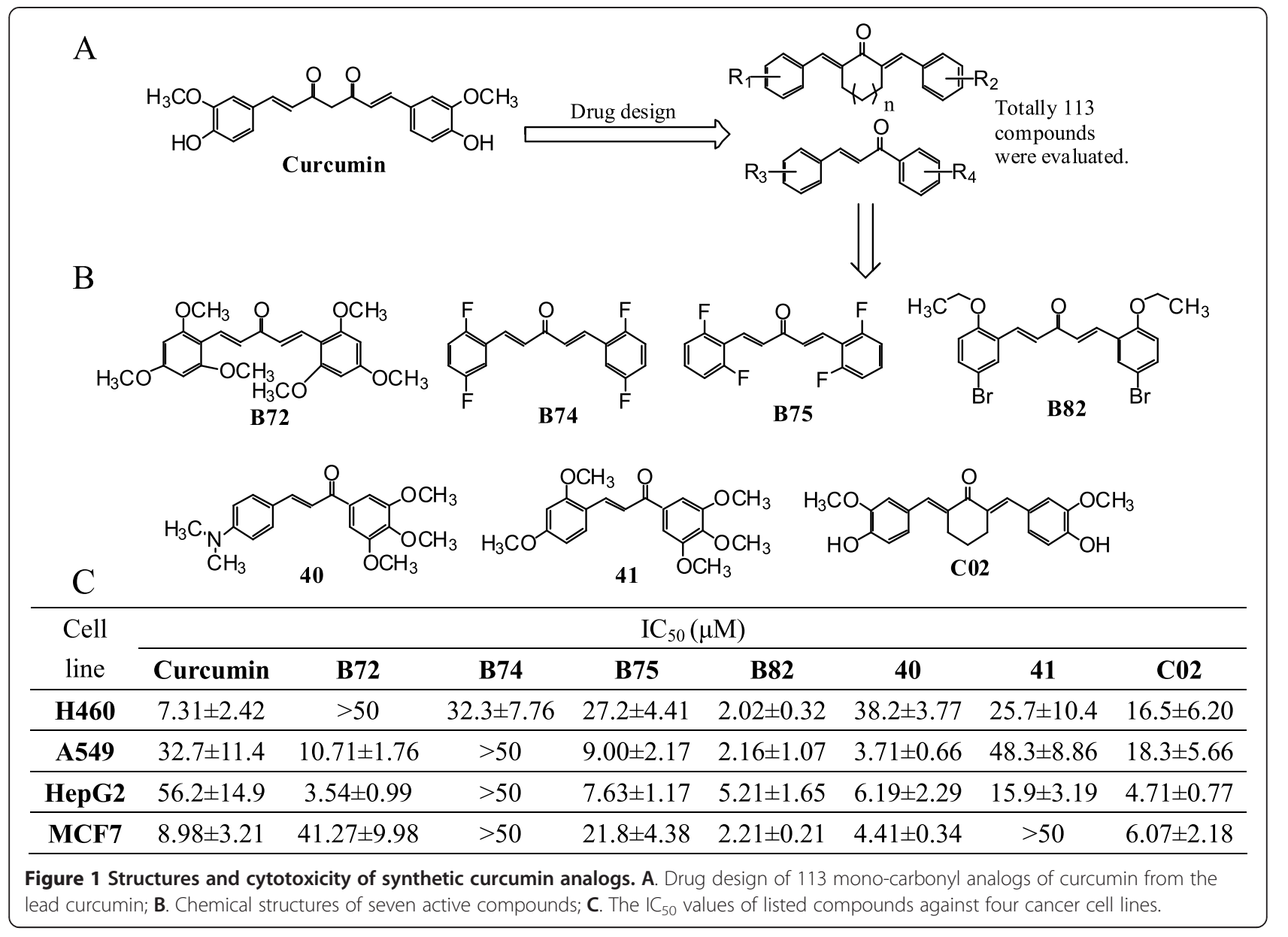

substituent, indicating that the electron-donating modification may be pharmacologically favorable for the anti-tumor drug design of this kind of MACs.

\section{B82 inhibited proliferation and induced apoptosis in $\mathrm{H} 460$ cells}

Although our findings clearly show B82 to have anti-cancer properties, the mechanism involved is unknown. We extended our study to include cell proliferation in $\mathrm{H} 460$ cells. Inhibitory effects of B82 at 2.5 and $10 \mu \mathrm{M}$ on the proliferation of $\mathrm{H} 460$ cells on laminin-coated plates were tested using the RT-CES system. As shown in Figure 2A, B82 treatment strongly suppressed the proliferation of H460 cells. The RT-CES assay is a convenient way to continuously determine cell number and cell activity and offers a full-range detection of B82-induced effects. Figure 2A showed a continuously change of H460 cells during $0 \mathrm{~h}-56 \mathrm{~h}$ after B82 treatment. At about $12 \mathrm{~h}$ after B82 addition, H460 cells were undergoing death or apoptosis. We next assessed the effect of B82 on the induction of apoptosis in H460 cells by flow cytometry. Figure 2B and $2 \mathrm{C}$ show that B82 dose-dependently increased $\mathrm{H} 460$ apoptosis after 12 -h treatment. B82 at $10 \mu \mathrm{M}$ induced a higher cell apoptosis rate (Annexin $\mathrm{V}^{+} / \mathrm{PI}^{-}, 15.33 \pm 2.96 \%$ ) than that of curcumin $(6.667 \pm 0.88 \%)$. Human lung (bronchial) epithelial cell line, BEAS-2B, was used to determine whether B82 has effects on normal lung cells. Our data found that it showed much higher $\mathrm{IC}_{50}$ values toward BEAS-2B cells than H460 cells (Figure 2D), indicating possible anti-cancer selectivity and safety. In addition, B82 showed low cytotoxicity against human normal lung epithelial cell line MRC-5 $\left(\mathrm{IC}_{50}=33.59 \mu \mathrm{M}\right)$ and normal human liver cell line HL-7702 $\left(\mathrm{IC}_{50}=37.86 \mu \mathrm{M}\right)$.

\section{B82 activated ER stress-mediated apoptotic pathway}

CHOP is considered as a marker of commitment of ER stress-mediated apoptosis [14]. We examined the effect of B82 on CHOP expression in H460 and HepG2 cells. As shown in Figure 3A-B, B82 is able to dose-dependently stimulate CHOP expression in both cell lines after 12-h treatment, indicating an evident activation of ER stress by B82 treatment. In comparison, B82 at the same concentrations could not induce the expression of $\mathrm{CHOP}$ in BEAS$2 \mathrm{~B}$ cells (Figure $3 \mathrm{C}$ ), suggesting a selectivity towards cancer cells of B82 activating ER stress. Glucose-regulate protein/ immunoglobulin heavy chain binding protein (GRP78) is 


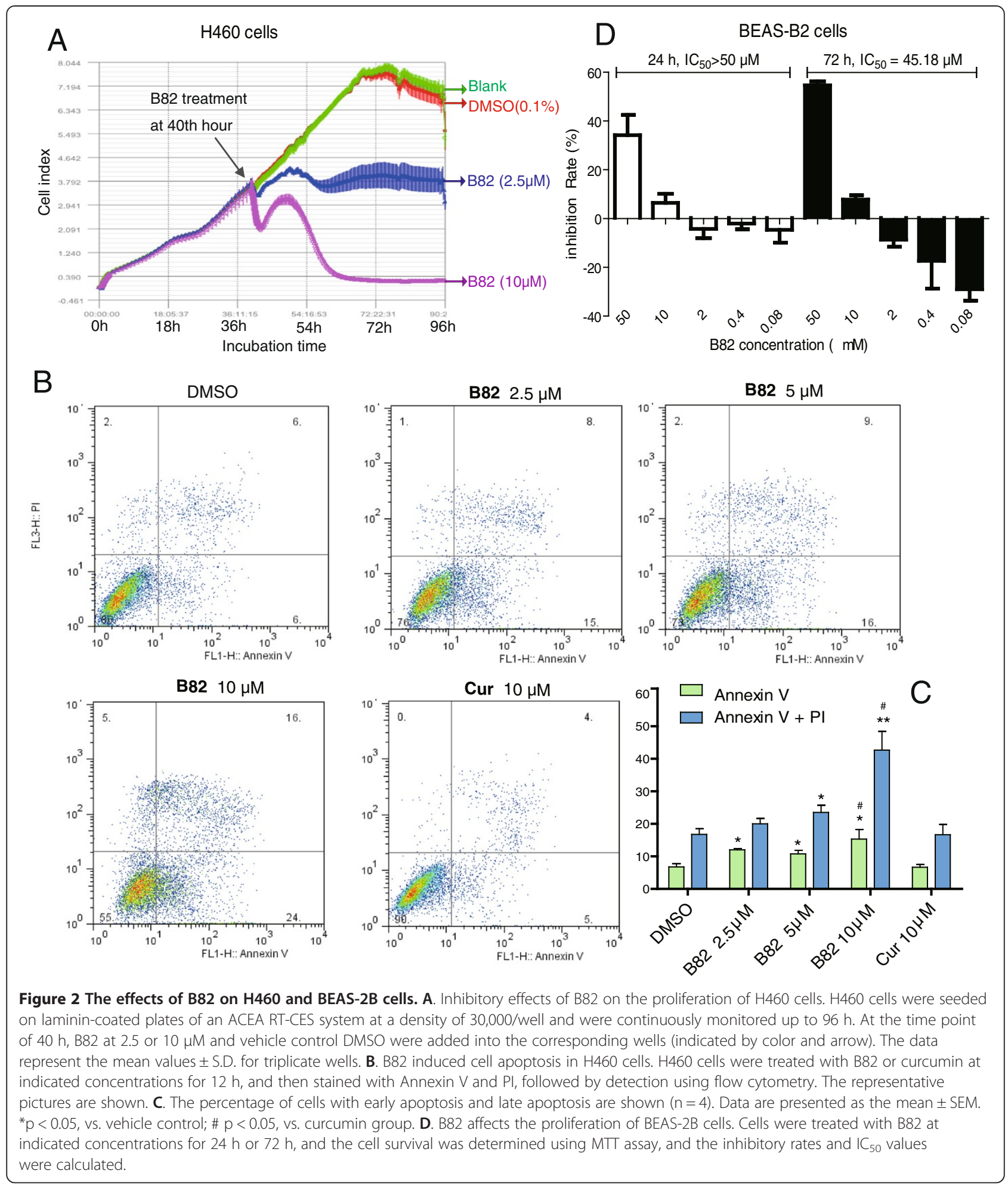

reported as the gatekeeper to the activation of the ER stress [15]. As shown in Figure 3D, treatment with B82 for $6 \mathrm{~h}$ significantly increased GRP78 mRNA level in H460 cells in a dose-dependent manner. Followed the gatekeeper, we tested the expressions of the downstream activating transcription factor 4 (ATF-4) and X-box binding protins-1 (XBP-1) in B82- or vehicle-treated H460 cells. RT-qPCR analysis revealed the significant increases in mRNA expression of both ATF-4 (Figure 3E) and XBP-1 (Figure 3F) in $\mathrm{H} 460$ cells after 6 -h treatment with $\mathrm{B} 82$. We also 


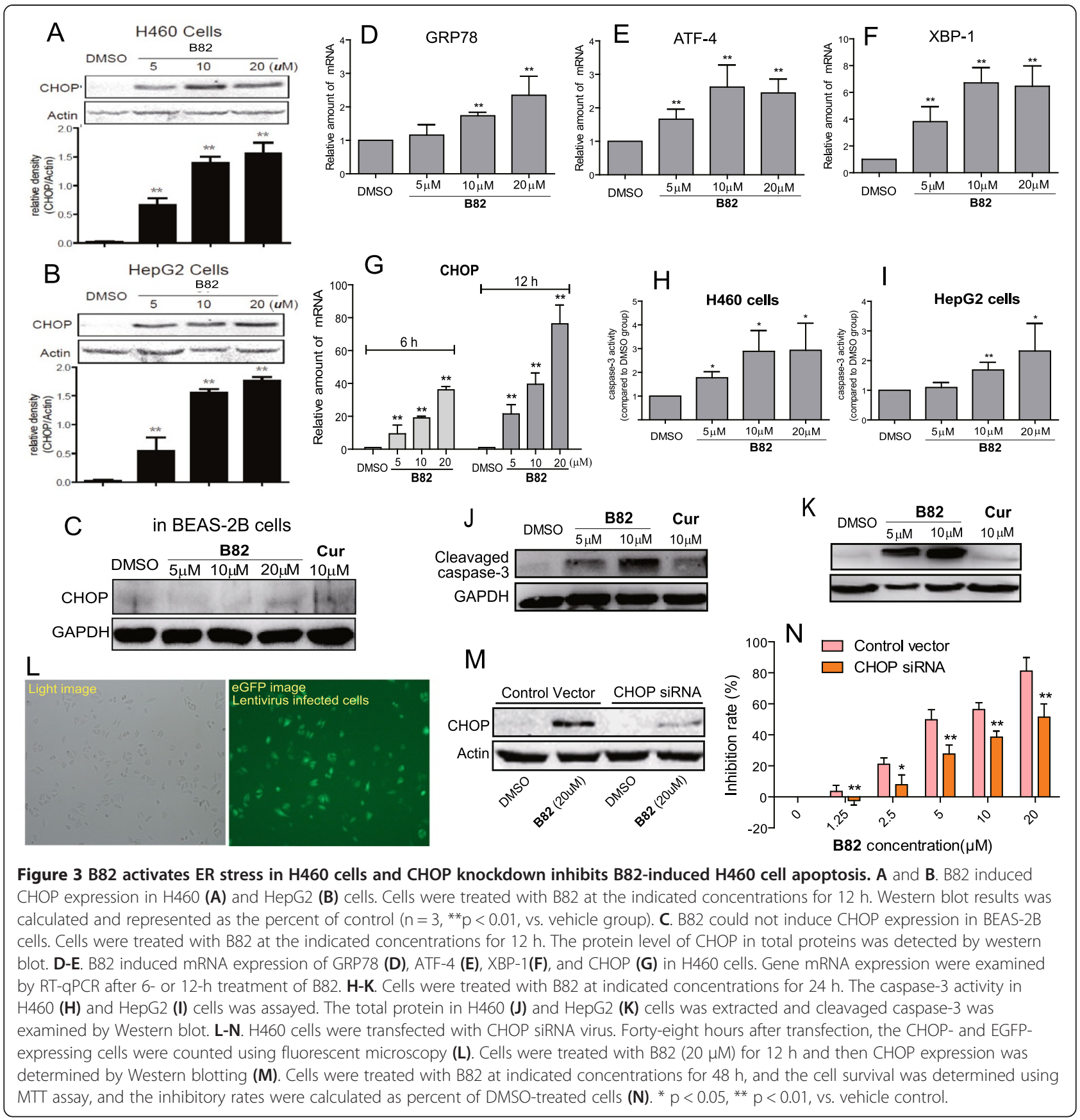

confirmed the effect of B82 in induction of CHOP mRNA expression. Figure $3 \mathrm{G}$ gives the time course result showing that treatment with B82 for $12 \mathrm{~h}$ led to a more significant increase in CHOP mRNA expression compared to 6-h treatment.

Regarding to the structural feature in molecules inducing ER stress, B82 possesses two electron-donating substituents (ethoxyl group) in 2'-position of the benzene rings. These two ethoxy groups in B82 structure could increase the electrophilicity of the central $\alpha, \beta$-unsaturated ketone as a Michael receptor. This supports the hypothesis that the cellular Michael adduct formation is responsible for ER stress activation via disrupting disulfide bond formation and consequently causing accumulation of unfolded proteins by the electrophilic olefine ketone [16]. These results are favorable for the anti-cancer drug design from curcumin analogs.

All upstream signals ultimately lead to caspase-3 activation to finish the execution of ER stress-induced apoptosis. The enzymatic activity of caspase- 3 induced by B82 was assayed in both H460 (Figure 3H) and HepG2 (Figure 3I) cells. The activity of caspase- 3 was increased in both cell 
lines after 24-h treatment with B82, in a dose-dependent way. Similar results were observed in the detection of cleavaged caspase- 3 . The cleavaged caspase- 3 was significantly increased by the treatment with B82 for $24 \mathrm{~h}$ in both $\mathrm{H} 460$ (Figure 3J) and HepG2 (Figure 3K) cells, while curcumin at $10 \mu \mathrm{M}$ could not activate caspase-3. In addition, other apoptotic markers, including cleaved-PARP (a downstream protein of caspase-3), P53, and Bax, were detected in B82-treated $\mathrm{H} 460$ cells by western blot method. As shown in Additional file 1: Figure S1, B82 at $10 \mu \mathrm{M}$ could increase the expression of these three proteins after $24 \mathrm{~h}$ treatment. These data confirmed that B82 treatment induced apoptosis in H460 cells.

\section{Reduction of CHOP expression inhibits B82-induced H460 cell death}

In order to further confirm that ER stress plays a critical role in the induction of $\mathrm{H} 460$ apoptosis by B82, we constructed the lentiviral siRNA for CHOP gene, which encoded the CMV-promoted EGFP marker as an internal control. As shown in Figure 3L, more than $75 \%$ of the
H460 cells were transfected with lentiviral siRNA. Furthermore, the reduction of CHOP expression was confirmed by western blot assay in Figure 3M, showing that $\mathrm{CHOP}$-siRNA significantly reduced B82-induced CHOP expression compared to the vector-transfected control. Finally, we treated CHOP siRNA-transfected $\mathrm{H} 460$ cells with B82 at indicated concentrations. Figure 3N shows that silencing CHOP expression in $\mathrm{H} 460$ cells significantly inhibited the cell apoptosis induced by B82, with a 4.14-fold increase in $\mathrm{IC}_{50}$ value from $4.63 \mu \mathrm{M}$ to $19.17 \mu \mathrm{M}\left({ }^{*} \mathrm{P}<0.05\right.$, ** $\left.\mathrm{P}<0.01\right)$. These data demonstrate that B82-induced cell apoptosis is, at least partly, mediated by $\mathrm{CHOP}$. However, other apoptotic mechanisms may also be involved in B82-induced apoptosis. The leading curcumin has been reported to exert anticancer effects by multitargeting mechanisms [2-5]. Despite that the siRNA CHOP could not completely wipe out ER stress activation, it only partly attenuated the apoptosis in H460 cells. Therefore, although this work only focuses on the ER stress-mediated apoptosis, further studies are necessary to establish such notions.

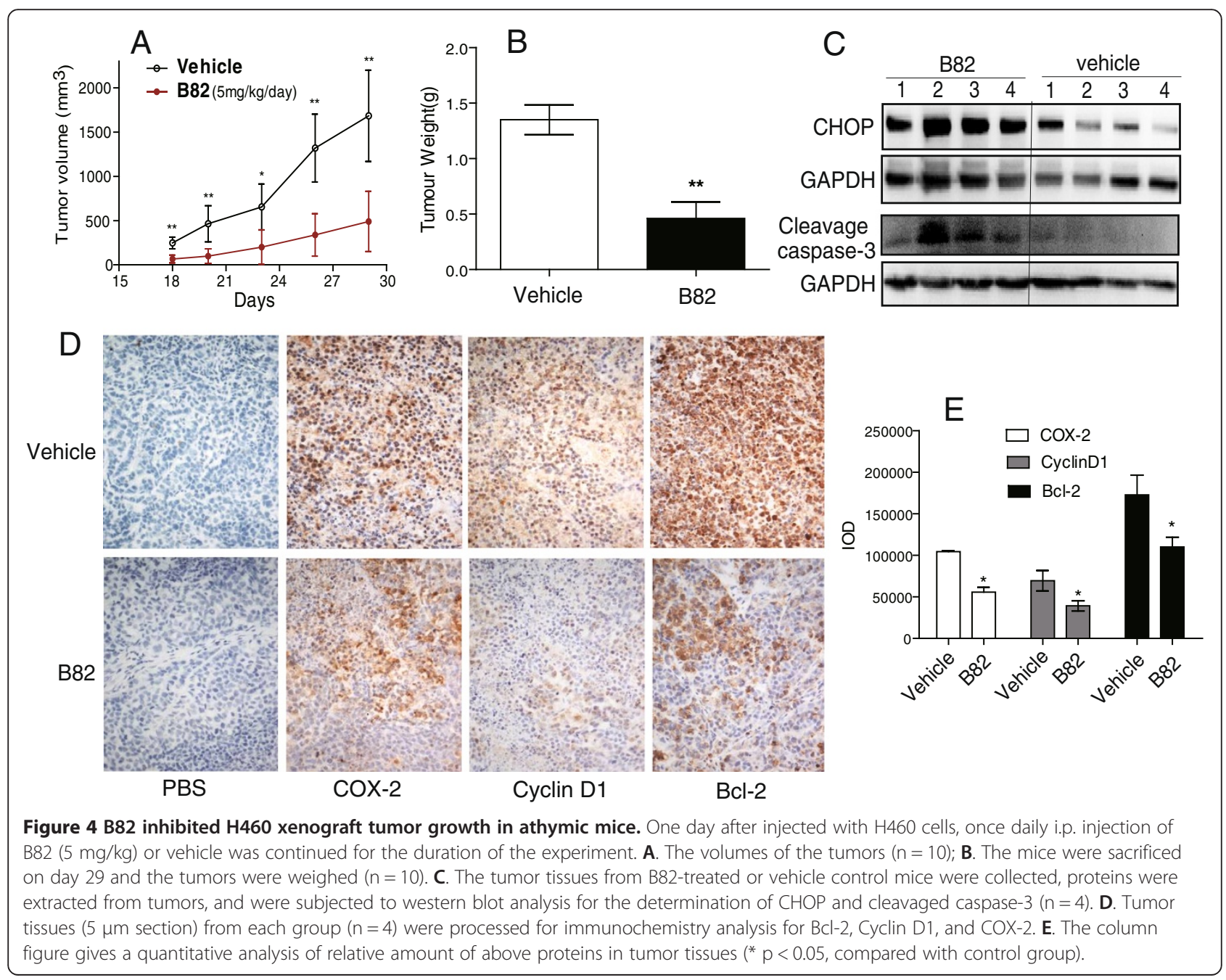




\section{B82 inhibited $\mathrm{H} 460$ tumor growth in vivo}

We further investigated the in vivo anti-tumor effect of B82 using BALB/c nude mouse. A water-soluble preparation of B82 was prepared using a patented liposome technique as described in our previous paper [17]. Continuous once daily i.p. administration of mice with B82 at $5 \mathrm{mg} / \mathrm{kg}$ resulted in a significant inhibition of $\mathrm{H} 460$ xenografts tumor growth compared to that observed in vehicle group (Figure 4A). A reduction of tumor weight by B82 administration with treated versus control $(\mathrm{T} / \mathrm{C})$ of $65.9 \%$ was observed on day 28 after treatment (Figure 4B). B82 was well tolerated with no obvious weight loss over the treatment period, suggesting that it is relatively nontoxic to mice (data not shown).

To confirm the anti-tumor mechanism of B82 in vivo, we harvested the tumor tissues after the 28-day treatment. As shown in Figure 4C, CHOP expression was significantly increased in the B82-treated group compared to the vehicle control group. Similar results were observed in the expression of cleavaged caspase-3, indicating that the in vivo anti-tumor effect of B82 is also associated with ER-stress-mediated apoptosis.

In addition, we also detected other signaling markers possibly involved in the anti-tumor action of B82. The relative number of $\mathrm{Bcl}-2$, cyclinD1, and $\mathrm{COX}-2$ positive tumor cells was substantially less in tumors from mice treated with B82, when compared with control tumors (Figure 4D). Quantification of these stained samples showed 1.4- to 2-fold decreases in the number of oncogene-positive cells in the B82-treated groups compared with the control group (Figure 4E). Bcl-2 and COX-2 are identified as oncoproteins and play important roles in the mitochondriamediated apoptotic pathway [18]. Cyclin D1 is a key protein in cell proliferation and is required for cell cycle G1/S transition. These results are also consistent with Figure $3 \mathrm{~N}$, in which silencing CHOP only partly attenuated the apoptosis in $\mathrm{H} 460$ cells. Thus, other apoptotic mechanisms may be also involved in B82-induced apoptosis that need be further investigated.

\section{Conclusions}

In summary, a new monocarbonyl analog of curcumin, B82, was shown to exhibit anti-tumor effects on NSCLC via an ER stress-mediated mechanism. Although a series of curcumin analogs have been reported to exert anticancer effects both in vitro and in vivo, the molecular mechanism of these compounds are still unclear, and like curcumin, a majority of them showed multi-targeting mechanisms. The discovery of activation of ER stresss-mediated apoptosis by curcumin analog B82 may provide new strategy for curcumin-based anticancer drug design and development. In addition, we note that B82 also shows an excellent antiinflammatory activity, and inhibits LPS-induced TNF- $\alpha$ and IL- 6 release in mouse macrophages [10]. Further investigation should demonstrate the possible crosstalk and complementation between its anti-inflammation and anti-tumor properties. The new compound B82 could be further explored as a potential anticancer agent for the treatment of NSCLC.

\section{Additional file}

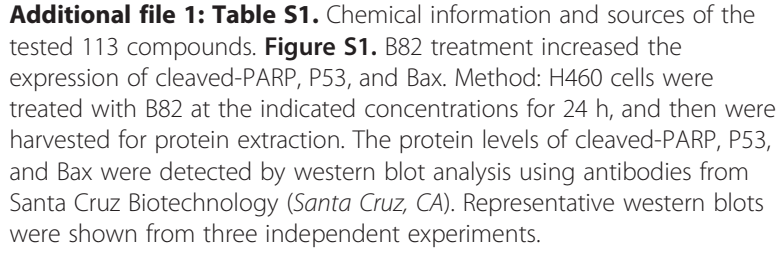

Additional file 1: Table S1. Chemical information and sources of the tested 113 compounds. Figure S1. B82 treatment increased the expression of cleaved-PARP, P53, and Bax. Method: H460 cells were treated with B82 at the indicated concentrations for $24 \mathrm{~h}$, and then were harvested for protein extraction. The protein levels of cleaved-PARP, P53, and Bax were detected by western blot analysis using antibodies from Santa Cruz Biotechnology (Santa Cruz, CA). Representative western blots were shown from three independent experiments.

\section{Abbreviations}

ER: Endoplasmic reticulum; MCACs: Mono-carbonyl analogs of curcumin; UPR: Unfolded protein response; NSCLC: Non small cell lung cancer; CHOP: C/EBP-homologous protein/growth arrest and DNA damage-inducible gene 153; GRP78: Glucose-regulate protein 78; ATF-4: Activating transcription factor 4; XBP-1: X-box binding proteins-1.

\section{Competing interests}

The authors declare that they no competing interest.

\section{Authors' contributions}

ZL carried out experiments, and performed data analysis. YS, LR, QW, XS carried out experiments. $\mathrm{YH}$ and $\mathrm{YC}$ contributed to supply testing compounds. XL participated in research design. GL participated in research design, performed data analysis, and drafted the manuscript. YW participated in research design, conducted experiments, performed data analysis, and drafted the manuscript. All authors read and approved the final manuscript.

\section{Acknowledgments}

This study was supported in part by Natural Science Funding of China (21202124 to Z. Liu, 81102452 to Y. Wang, and 81173083 to Y. Cai), Natural Science Funding of Zhejiang (Y2110568 to Y. Sun), Zhejiang Health Science and Technology Project (2011RCB025 to Y.Wang and 2013KYB168 to Z. Liu), Zhejiang Key Health Science and Technology Project (WKJ2013-2-021 to G. Liang), High-level Inovative Talent Funding of Zhejiang Department of Health (to G. Liang), and Zhejiang Key Group in Scientific Innovation (2010R50042 to X.L.)

\section{Author details}

${ }^{1}$ Chemical Biology Research Center, School of Pharmaceutical Sciences, Wenzhou Medical University, University Town, 325035 Wenzhou, Zhejiang, China. ${ }^{2}$ Department of general surgery, the First Affiliated Hospital of Wenzhou Medical University, Wenzhou, Zhejiang, China.

Received: 26 March 2013 Accepted: 17 October 2013 Published: 24 October 2013

\section{References}

1. Wang $S$, Kaufman RJ: The impact of the unfolded protein response on human disease. J Cell Biol 2012, 197(7):857-867.

2. Sun SH, Huang HC, Huang C, Lin JK: Cycle arrest and apoptosis in MDA-MB-231/Her2 cells induced by curcumin. Eur J Pharmacol 2012, 690(1-3):22-30.

3. Ng AP, Chng WJ, Khan M: Curcumin sensitizes acute promyelocytic leukemia cells to unfolded protein response-induced apoptosis by blocking the loss of misfolded N-CoR protein. Mol Cancer Res 2011, 9(7):878-888.

4. Wu SH, Hang LW, Yang JS, Chen HY, Lin HY, Chiang JH, Lu CC, Yang JL, Lai TY, Ko YC, et al: Curcumin induces apoptosis in human non-small cell lung cancer $\mathrm{NCl}-\mathrm{H} 460$ cells through ER stress and caspase cascade- and mitochondria-dependent pathways. Anticancer Res 2010, 30(6):2125-2133. 
5. Wang L, Song R, Shen Y, Sun Y, Gu Y, Shu Y, Xu Q: Targeting sarcoplasmic/ endoplasmic reticulum $\mathrm{Ca}(2)+-$ ATPase 2 by curcumin induces ER stress-associated apoptosis for treating human liposarcoma. Mol Cancer Ther 2011, 10(3):461-471.

6. Anand P, Kunnumakkara AB, Newman RA, Aggarwal BB: Bioavailability of curcumin: problems and promises. Mol Pharm 2007, 4(6):807-818.

7. Liang G, Yang S, Zhou H, Shao L, Huang K, Xiao J, Huang Z, Li X: Synthesis, crystal structure and anti-inflammatory properties of curcumin analogues. Eur J Med Chem 2009, 44(2):915-919.

8. Liang G, Zhou H, Wang Y, Gurley EC, Feng B, Chen L, Xiao J, Yang S, Li X: Inhibition of LPS-induced production of inflammatory factors in the macrophages by mono-carbonyl analogues of curcumin. J Cell Mol Med 2009, 13(9B):3370-3379.

9. Zhao C, Yang J, Wang Y, Liang D, Yang X, Li X, Wu J, Wu X, Yang S, Liang G: Synthesis of mono-carbonyl analogues of curcumin and their effects on inhibition of cytokine release in LPS-stimulated RAW 264.7 macrophages. Bioorg Med Chem 2010, 18(7):2388-2393.

10. Zhao C, Cai Y, He X, Li J, Zhang L, Wu J, Zhao Y, Yang S, Li X, Li W, et al: Synthesis and anti-inflammatory evaluation of novel mono-carbonyl analogues of curcumin in LPS-stimulated RAW 264.7 macrophages. Eur J Med Chem 2010, 45(12):5773-5780.

11. Wu J, Li J, Cai Y, Pan Y, Ye F, Zhang Y, Zhao Y, Yang S, Li X, Liang G: Evaluation and discovery of novel synthetic chalcone derivatives as anti-inflammatory agents. J Med Chem 2011, 54(23):8110-8123.

12. Liang G, Li X, Chen L, Yang S, Wu X, Studer E, Gurley E, Hylemon PB, Ye F, $\mathrm{Li} Y$, et al: Synthesis and anti-inflammatory activities of mono-carbonyl analogues of curcumin. Bioorg Med Chem Lett 2008, 18(4):1525-1529.

13. Liang G, Shao LL, Wang Y, Zhao CG, Chu YH, Xiao J, Zhao Y, Li XK, Yang SL: Exploration and synthesis of curcumin analogues with improved structural stability both in vitro and in vivo as cytotoxic agents. Bioorg Med Chem 2009, 17(6):2623-2631.

14. Han J, Murthy R, Wood B, Song B, Wang S, Sun B, Malhi H, Kaufman RJ: ER stress signalling through elF2alpha and CHOP, but not IRE1alpha, attenuates adipogenesis in mice. Diabetologia 2013, 56(4):911-924.

15. Hammadi M, Oulidi A, Gackiere F, Katsogiannou M, Slomianny C, Roudbaraki M, Dewailly E, Delcourt P, Lepage G, Lotteau S, et al: Modulation of ER stress and apoptosis by endoplasmic reticulum calcium leak via translocon during unfolded protein response: involvement of GRP78. FASEB J 2013.

16. Wang X, Thomas B, Sachdeva R, Arterburn L, Frye L, Hatcher PG, Cornwell $D G$, Ma J: Mechanism of arylating quinone toxicity involving Michael adduct formation and induction of endoplasmic reticulum stress. Proc Natl Acad Sci U S A 2006, 103(10):3604-3609.

17. Wang Y, Xiao J, Zhou H, Yang S, Wu X, Jiang C, Zhao Y, Liang D, Li X, Liang G: A Novel Monocarbonyl Analogue of Curcumin, (1E,4E)-1,5-Bis (2,3-dimethoxyphenyl)penta-1,4-dien-3-one, Induced Cancer Cell H460 apoptosis via activation of endoplasmic reticulum stress signaling pathway. J Med Chem 2011, 54(11):3768-3778.

18. Kilbride SM, Prehn JH: Central roles of apoptotic proteins in mitochondrial function. Oncogene 2013, 32(22):2703-2711.

doi:10.1186/1471-2407-13-494

Cite this article as: Liu et al:: Evaluation of a curcumin analog as an anti-cancer agent inducing ER stress-mediated apoptosis in non-small cell lung cancer cells. BMC Cancer 2013 13:494.

\section{Submit your next manuscript to BioMed Central and take full advantage of:}

- Convenient online submission

- Thorough peer review

- No space constraints or color figure charges

- Immediate publication on acceptance

- Inclusion in PubMed, CAS, Scopus and Google Scholar

- Research which is freely available for redistribution

Submit your manuscript at www.biomedcentral.com/submit
Ciomed Central 